\title{
The magnetic field in the photospheric and circumstellar components of Herbig Ae stars ${ }^{\star}$
}

\author{
S. Hubrig ${ }^{1}$, M. A. Pogodin ${ }^{2,3}$, R. V. Yudin ${ }^{2,3}$, M. Schöller ${ }^{1}$, and R. S. Schnerr ${ }^{4}$ \\ 1 European Southern Observatory, Casilla 19001, Santiago 19, Chile \\ e-mail: shubrig@eso.org \\ 2 Pulkovo Observatory, Saint-Petersburg, 196140, Russia \\ Isaac Newton Institute of Chile, Saint-Petersburg Branch, Russia \\ 4 Astronomical Institute "Anton Pannekoek", University of Amsterdam, Kruislaan 403, 1098 SJ Amsterdam, The Netherlands
}

Received 24 July 2006 / Accepted 12 October 2006

\section{ABSTRACT}

\begin{abstract}
Aims. We investigate the photospheric and circumstellar (CS) magnetic field components separately in seven Herbig Ae stars. Methods. The study is based on low-resolution $(R \sim 2000$ and 4000) spectropolarimetric data collected from 2003 to 2005 at the Very Large Telescope (ESO, Chile) with the multi-mode instrument FORS1.

Results. We show that the spectropolarimetric results strongly depend on the level of CS contribution to the stellar spectra. We have improved the determination accuracy of magnetic fields up to the $7 \sigma$ level in the two Herbig Ae stars HD 139614 and HD 144432 , observed in 2005 when these objects were at a low level state in their CS activity. We have established that at a higher level state of CS activity the polarisation signatures are mainly related to the CS matter. The presence of CS polarisation signatures formed in the stellar wind supports the assumption that the magnetic centrifuge is one of the main mechanisms of wind acceleration.

Conclusions. We conclude that the most effective way to investigate the magnetism of Herbig Ae stars is to monitor their spectropolarimetric behaviour at different stages of CS activity. Obviously, higher-resolution spectropolarimetric observations would extend the sample of spectral lines which can be used to measure magnetic fields at different levels in the stellar atmosphere and CS envelope. These observations will give a more complete insight into the magnetic topology in Herbig Ae stars.
\end{abstract}

Key words. stars: pre-main sequence - polarization - stars: magnetic fields - stars: circumstellar matter

\section{Introduction}

The Herbig Ae/Be stars (HAEBEs) are pre-main sequence (PMS) objects with masses of 1.5 to $10 M_{\odot}$ (Herbig 1960; Finkenzeller \& Mundt 1984; Thé et al. 1994). In their spectra, they frequently reveal a complex structure of the circumstellar (CS) material surrounding the star. According to common views, the envelopes of HAEBEs contain an equatorial gaseous-dusty disk and a stellar wind at higher latitudes. The matter outflow from HAEBEs is time-variable and spatially inhomogeneous, and signatures of a non-stable matter infall onto the star are frequently observed. Presently, there is a clear gap in our knowledge of the relation between these different processes.

Numerous theoretical works predict the existence of a global magnetic field of a complex configuration formed as a result of the interaction between the star and the accretion disk. Very likely, such a field is responsible for all observational peculiarities of HAEBEs. For this reason, an investigation of the strength and the topology of the magnetic field can provide important information on the origin of activity in HAEBEs.

Over an extended period of years, all attempts to obtain reliable, direct measurements of the magnetic fields of HAEBEs have been rather unsuccessful. On the one hand, the lack of field detections could be plausibly explained by the weakness of the

* Based on observations collected at the European Southern Observatory, Paranal, Chile (ESO programme Nos. 072.D-0377, 073.D0464, 074.C-0463, and 075.D-0507). magnetic fields in HAEBEs with strengths considerably lower than those in magnetic A-type stars or low-mass PMS T Tauri objects. In addition, the accuracy of all previous measurements was not good enough to detect such weak fields (Catala et al. 1993; Glagolevskij \& Chountonov 2001). On the other hand, the observational strategy only allowed the average value of the magnetic field to be determined using a large number of different spectral lines (Glagolevskij \& Chountonov 2001). Due to the very likely complex spatial structure of the magnetic fields of HAEBEs, the measured magnetic field strength is expected to strongly depend on the individual spectral lines used for the field determination. The measured magnetic field is probably varying significantly from the stellar atmosphere to different CS regions, even with a change in polarity. Undoubtedly, any attempt to detect and to study fields in HAEBEs must aim at achieving a considerably better accuracy of measurements and in applying a specific data analysis, which we describe in this paper.

The first detection of a magnetic field in a Herbig Ae star was obtained by Donati et al. (1997). A marginal circular polarisation signature indicating the existence of a weak magnetic field (about 50 G) was observed in metal lines of HD 104237. However, numerous observations carried out in the past years in various wavelength regions have revealed that HD 104237 is a multiple system containing four T Tauri-type components along with the Herbig A4-A7e star. The most massive companion has a bolometric luminosity that is only a factor of 10 less than the Herbig Ae primary (Feigelson et al. 2003; 
Böhm et al. 2004). Therefore, the possibility that the detected magnetic field of HD 104237 is actually related to the T Tauritype components (Guenter 1997) cannot be ruled out.

Quite recently, definite evidence of the presence of magnetic fields of the order of about $100 \mathrm{G}$ was presented for several Herbig Ae stars by Hubrig et al. (2004, 2006). For the measurements of the magnetic fields, we used spectropolarimetric observations obtained with the $8 \mathrm{~m}$ VLT + FORS 1 in the years 2003-2005. The accuracy of the field determinations was about $30 \mathrm{G}$ using exclusively Balmer lines in the spectropolarimetric data obtained at a spectral resolving power $R \sim 4000$. In these studies we reported $3 \sigma$ detections for three out of the seven Herbig Ae stars studied. Wade et al. (2006) carried out a re-analysis of the observational data for HD 139614 obtained by Hubrig et al. in 2003. They confirmed the detection of the mean longitudinal field in Balmer lines by measuring a field $\left\langle B_{z}\right\rangle=-320 \pm 75 \mathrm{G}$, which is similar to our former result $\left\langle B_{z}\right\rangle=-450 \pm 93 \mathrm{G}$. Curiously, the magnetic field was not detected when they also included numerous metal lines in their analysis.

The presence of a weak magnetic field in HD 139614 has been confirmed by our more recent spectropolarimetric observations in $2005\left(\left\langle B_{z}\right\rangle=-116 \pm 34 \mathrm{G}\right.$; Hubrig et al. 2006). Three weeks after our observations, Wade et al. (2006) used ESPaDOnS at the CFHT to obtain two additional spectropolarimetric observations of HD 139614. For the measurements of the magnetic field, the authors utilised metal lines using the leastsquares deconvolution method (LSD; Donati et al. 1997). No Zeeman signature has been detected in their mean circular polarisation profiles (LSD Stokes $V$ profiles). The non-detection of the magnetic field in HD 139614 is probably related to an inappropriate line list used by these authors that included not only photospheric absorption lines but also spectral lines showing emission and P Cygni profiles from the wind and/or circumstellar environment.

Longitudinal magnetic field measurements are only sensitive to the line-of-sight component of the magnetic field, $\left\langle B_{z}\right\rangle$. This method is most effective when lines form over a volume where the field varies slowly with radius, such as an atmosphere where scale heights set the scale for line formation. The resulting $\left\langle B_{z}\right\rangle$ is the average of the line of sight component of the field over the stellar hemisphere visible at the time of observations, weighted by the local emergent spectral line intensity. In a circumstellar environment, such as a wind or an accretion disk, lines may form over a relatively large volume, and the field topology may be complex not only in latitude and azimuth, but in radius as well. Thus, for complex magnetohydrodynamical flows in the presence of photospheric and circumstellar fields of comparable strength, it is quite easy to drive the net line-of-sight magnetic flux to near zero values.

Other possibilities for explaining the discrepancy between the different authors could be the expected variability of the field strength resulting from the variable CS contribution or the rotational modulation of the magnetic field, provided this star represents an inclined magnetic rotator. Even in the case where the photospheric fields are stronger than circumstellar fields, the presence of wind line emission would produce diminishing of the circular polarisation signal, i.e. polarimetric dilution caused by contributing unpolarised emission. If both photospheric and circumstellar contributions are present and an unparsed ensemble of lines is used, null detections may result because of the issues described here.

Our previous magnetic field measurements of seven Herbig Ae stars were carried out exclusively in the broad hydrogen lines (Hubrig et al. 2004, 2006). In the new analysis we have used the full spectrum, excluding only the regions that are contaminated by the circumstellar environment. Below, we report our results from analysing the magnetic fields in both the atmosphere and the circumstellar environment of this Herbig star sample. We have also included in this analysis two new spectropolarimetric observations of the Herbig Ae star HD 190073 and one observation of the Herbig Ae star HD 144668, all obtained in 2005. Considerable attention has been paid to the presentation of the circular polarisation spectra to demonstrate the Zeeman features indicating the presence of magnetic fields.

\section{Program targets and analysis strategy}

To analyse photospheric and circumstellar magnetic-field components in Herbig Ae stars, we used spectropolarimetric observations of seven Herbig Ae stars obtained in 2003-2005 with the multi-mode instrument FORS 1 installed at the $8 \mathrm{~m}$-KUEYEN telescope at the VLT (ESO, Chile). The circular polarisation measurements carried out in the wings of Balmer lines have already been reported by Hubrig et al. (2004, 2006). Two more observations of the Herbig Ae star HD 190073 were obtained at the end of May and in August 2005. One additional observation of the Herbig Ae star HD 144668 was obtained in April 2005. These have also been included in the present analysis.

FORS 1 is equipped with polarisation analysing optics comprising super-achromatic half-wave and quarter-wave phaseretarder plates and a Wollaston prism with a beam divergence of $22^{\prime \prime}$ in standard resolution mode. We used the GRISM 600B to cover all $\mathrm{H}$ Balmer lines from $\mathrm{H} \beta$ to the Balmer jump and GRISM $1200 \mathrm{~g}$ to cover the $\mathrm{H}$ Balmer lines from $\mathrm{H} \beta$ to $\mathrm{H} 8$. The spectral resolution of the FORS 1 spectra obtained with GRISM 600B is about 2000, whereas GRISM 1200g allows us to achieve a spectral resolving power of $R \sim 4000$ with the narrowest available slit width of 0.4 . The spectral regions covered by GRISM 600B and GRISM $1200 \mathrm{~g}$ are respectively $\lambda \lambda 3700-5900 \AA$ and $\lambda \lambda 3900-5000 \AA$. A detailed description of the assessment of the longitudinal magnetic field measurements using the FORS 1 spectra is presented in our previous papers (Hubrig et al. 2004, 2006) and in the references therein.

The technique applied for measuring magnetic fields in stellar atmospheres was developed by Angel \& Landstreet (1970), allowing determination of the mean longitudinal magnetic field $\left\langle B_{z}\right\rangle$ of the star. It works well if the line profiles are axially symmetric and if the Zeeman splitting is small compared to the intrinsic broadening of the line (so-called weak-field regime). However, their technique cannot be applied to spectral lines originating in the CS material, because profiles of these spectral lines are mainly determined by the velocity field in the CS gas and are seldom axially symmetric. The motion of CS gas is expected to be strongly governed by a magnetic field, especially in the regions of the stellar wind, which is generally assumed to be accelerated by a magnetic centrifuge. Therefore, different parts of individual line profiles can diagnose CS regions with very different strengths of the magnetic field. Nevertheless, circular polarisation signatures observed at the wavelengths corresponding to clearly identified CS lines allow the study of a magnetic field in the region of the line formation. If such circular polarisation features are indeed observed, one can infer the presence of a magnetic field. However, numerical determination of $\left\langle B_{z}\right\rangle$ in the CS region can only be carried out by using a special method.

The goals of our study were as follows: a) to analyse the spectropolarimetric data of seven programme stars and to 
Table 1. Atmospheric parameters used for calculating the synthetic photospheric spectra of the programme targets.

\begin{tabular}{cccc}
\hline \hline $\mathrm{HD}$ & $T_{\mathrm{eff}}(\mathrm{K})$ & $\log g$ & $v \sin i$ \\
\hline 31648 & 9250 & 3.5 & 90 \\
38238 & 7750 & 3.5 & 100 \\
139614 & 8250 & 4.0 & 15 \\
144432 & 7250 & 4.0 & 70 \\
144668 & 7500 & 4.0 & 100 \\
163296 & 9250 & 3.5 & 130 \\
190073 & 9250 & 4.0 & 12 \\
\hline
\end{tabular}

separate the photospheric and CS components of all spectral lines; b) to re-measure the photospheric magnetic fields of Herbig Ae stars without inclusion of spectral regions affected by the CS matter; c) to derive information on the presence of magnetic fields in the CS environment. The idea of detecting the Zeeman effect in the CS environment is rather new. The first theoretical assessment of wind signatures in Stokes $V$ profiles has been presented by Ignace \& Gayley (2003).

To determine the spectral region affected by the CS matter, we have compared the observed spectra for each star with synthetic photospheric spectra computed for models of stellar atmospheres with the corresponding stellar parameters $T_{\text {eff }}$ and $\log g$. The synthetic photospheric spectra were calculated with the computer code SYNTH + ROTATE developed by Piskunov (1992). Because of the rather low resolution of our spectra, the fitting was only applied to slopes of wide Starkbroadened wings of the Balmer $\mathrm{H} \beta, \mathrm{H} \gamma, \mathrm{H} \delta$, and $\mathrm{H} \epsilon$ lines. The results of our assessment of atmospheric parameters are presented in Table 1 along with the values of $v \sin i$, which were determined from high-resolution non-polarised spectra collected in recent years with EMMI at the NTT on La Silla and at the Crimean Observatory.

\section{Results}

We suggest that the observations of the studied Herbig Ae stars can be divided into three groups. The first group contains objects with photospheric magnetic fields that have been detected at a high confidence level of about $7 \sigma$. The objects with only weak circular polarisation signatures, which however cannot be determined with sufficient precision, constitute the second group. Objects with clear circular polarisation signatures mainly of a $\mathrm{CS}$ origin have been distinguished as a third group. The magnetic field has been remeasured exclusively in spectral regions not contaminated by the CS material.

The results of our analysis are summarised in Table 2. Each row corresponds to one measurement. In the first column we identify the group a measurement falls in. The second column gives the name of the target, the third column the modified Julian date of the observation, and the fourth column the spectral resolution of the measurement. In column five we list the value for $\left\langle B_{z}\right\rangle$ from the analysis in Hubrig et al. (2006), where we used all the Balmer lines, except for HD 190073, where we used only $\mathrm{Ca}$ II $\mathrm{H}+\mathrm{K}$. In column six we list the value for $\left\langle B_{z}\right\rangle$ from the analysis reported in this paper, using the same data. Here, we have taken the full spectrum, except for the Balmer lines and the Ca II lines, where we detect contamination of the lines by the CS environment. In the case where we detect the stellar magnetic field with a high confidence level, we indicate this in column seven by a $(+)$; in cases where we find a field but believe it is distorted by the CS material, we indicate this in column seven by a (?). In the last column we list the lines for which signatures of a CS magnetic field are clearly seen. In the following we discuss the results obtained for each group.

\subsection{Group I}

\subsubsection{HD 139614 (observed in 2005)}

The comparison of the spectrum of HD 139614 observed in 2005 with the synthetic photospheric spectrum reveals that the spectrum is mainly of photospheric origin. The only exceptions are the first members of the Balmer series. Broad profiles of these lines are overlapped by low-intensity central emissions whose strength drops gradually from $\mathrm{H} \beta$ to $\mathrm{H} \delta$. The spectrum of the object in the blue spectral region $\lambda \lambda 4200-4300 \AA$ contains plenty of metal lines. The Stokes $V$ spectrum in this region shows a noticeable correlation between the wavelength of many absorption lines and circular polarisation signatures (Fig. 1, top). The most prominent polarisation signatures are observed in the $\mathrm{H}$ and $\mathrm{K}$ components of the resonance $\mathrm{Ca}$ II doublet, as well as in the Fe I blend at $\lambda 3956.8 \AA$ (Fig. 1, bottom).

No polarisation signatures are observed in the $\mathrm{H} \beta, \mathrm{H} \gamma$, and $\mathrm{H} \delta$ lines. We explain this fact by a significant contribution of CS material distorting the circular polarisation signatures of the atmospheric component. Excluding these distorted spectral regions from the evaluation of the magnetic field, we determine a very accurate value for the magnetic field in this star $\left\langle B_{z}\right\rangle=-93 \pm 14 \mathrm{G}$.

\subsubsection{HD 144432 (observed in 2005)}

The spectrum of HD 144432 reveals signatures of a dense stellar wind, i.e. the wind emission is strong enough to appear in the lines. Blueshifted absorption components are clearly seen in $\mathrm{H} \beta$, $\mathrm{H} \gamma, \mathrm{H} \delta, \mathrm{H} \epsilon$, and the Ca II doublet lines. Strong circular polarisation signatures at the wavelengths of these lines indicate that outflowing gas in the wind region is magnetised (Fig. 2). Similar to HD 139614, HD 144432 shows noticeable polarisation signatures in metal lines. Excluding the spectral regions containing the hydrogen and Ca II doublet lines, we determine the photospheric magnetic field $\left\langle B_{z}\right\rangle=-111 \pm 16 \mathrm{G}$.

\subsection{Group II}

\subsubsection{HD 38238}

The CS magnetic field component in the spectral lines of HD 38238 is only weakly present (Fig. 3). Faint circular polarisation signatures are detectable in Balmer and Ca II lines, but they probably represent a combination of the stellar atmospheric and CS magnetic-field signatures. No magnetic field has been detected in this star.

\subsubsection{HD 139614 (observed in 2003)}

Previous spectropolarimetric observations of HD 139614 carried out in 2003 (Hubrig et al. 2004) showed a significantly higher level of CS activity in comparison with the 2005 data. Figure 4 presents the Stokes $V$ spectrum of HD139614 obtained near the Ca II doublet in 2003. The lower spectral resolution $(R \sim 2000)$ does not allow us to estimate the CS contribution to the stellar spectrum in comparison with the synthetic spectrum of the object very accurately. Nevertheless, we believe that the CS contribution in 2003 was significantly higher than in 2005. The 
Table 2. Results of the magnetic field measurements for each group.

\begin{tabular}{|c|c|c|c|c|c|c|c|}
\hline Group & HD & MJD & $R$ & $\begin{array}{c}\left\langle B_{z}\right\rangle \\
\text { previous }\end{array}$ & $\begin{array}{l}\left\langle B_{z}\right\rangle \\
\text { new }\end{array}$ & $\begin{array}{c}\text { Stellar } \\
\text { magnetic field }\end{array}$ & $\begin{array}{c}\mathrm{CS} \\
\text { magnetic field }\end{array}$ \\
\hline I & $\begin{array}{l}139614 \\
144432\end{array}$ & $\begin{array}{l}53405.37 \\
53447.35\end{array}$ & $\begin{array}{l}4000 \\
4000\end{array}$ & $\begin{array}{l}-116 \pm 34 \mathrm{G} \\
-119 \pm 38 \mathrm{G}\end{array}$ & $\begin{aligned}-93 \pm 14 G \\
-111 \pm 16 G\end{aligned}$ & $\begin{array}{l}+ \\
+\end{array}$ & $\mathrm{H} \beta-\mathrm{H} \epsilon, \mathrm{Ca}$ II $\mathrm{H}+\mathrm{K}$ \\
\hline II & $\begin{array}{l}38238 \\
139614 \\
144432 \\
144668 \\
144668 \\
144668\end{array}$ & $\begin{array}{l}53249.37 \\
52904.04 \\
52900.99 \\
52901.01 \\
53120.25 \\
53461.30\end{array}$ & $\begin{array}{l}2000 \\
2000 \\
2000 \\
2000 \\
2000 \\
4000\end{array}$ & $\begin{aligned} &-115 \pm 67 \mathrm{G} \\
&-450 \pm 93 \mathrm{G} \\
&-94 \pm 60 \mathrm{G} \\
&-118 \pm 48 \mathrm{G} \\
&-107 \pm 40 \mathrm{G} \\
&-\end{aligned}$ & $\begin{array}{r}+13 \pm 36 \mathrm{G} \\
-112 \pm 36 \mathrm{G} \\
+32 \pm 37 \mathrm{G} \\
+166 \pm 40 \mathrm{G} \\
-75 \pm 29 \mathrm{G} \\
+195 \pm 121 \mathrm{G}\end{array}$ & ? & $\begin{array}{c}\mathrm{H} \beta-\mathrm{H} \epsilon \text {, Сa II H+K } \\
\mathrm{H} \beta-\mathrm{H} \epsilon \text {, Ca II H+K } \\
\mathrm{H} \beta-\mathrm{H} \epsilon \text {, Сa II H+K } \\
\mathrm{H} \beta-\mathrm{H} 10, \text { Ca II H+K } \\
\mathrm{H} \beta-\mathrm{H} 10, \text { Ca II H+K } \\
\mathrm{H} \beta-\mathrm{H} 10, \text { Ca II H+K }\end{array}$ \\
\hline III & $\begin{array}{c}31648 \\
163296 \\
190073 \\
190073 \\
190073\end{array}$ & $\begin{array}{l}53296.35 \\
53279.00 \\
53514.36 \\
53519.38 \\
53596.15\end{array}$ & $\begin{array}{l}4000 \\
4000 \\
4000 \\
4000 \\
4000\end{array}$ & $\begin{array}{c}+87 \pm 22 \mathrm{G} \\
-57 \pm 33 \mathrm{G} \\
- \\
+84 \pm 30 \mathrm{G} \\
-\end{array}$ & $\begin{array}{l}+73 \pm 32 \mathrm{G} \\
-25 \pm 27 \mathrm{G} \\
+21 \pm 12 \mathrm{G} \\
+14 \pm 22 \mathrm{G} \\
-51 \pm 14 \mathrm{G}\end{array}$ & $?$ & $\begin{array}{c}\mathrm{H} \beta-\mathrm{H} \epsilon, \mathrm{Ca} \text { II H}+\mathrm{K} \\
\mathrm{Ca} \text { II H+K } \\
\mathrm{Ca} \text { II H+K } \\
\mathrm{Ca} \text { II H+K } \\
\mathrm{Ca} \text { II H+K }\end{array}$ \\
\hline
\end{tabular}
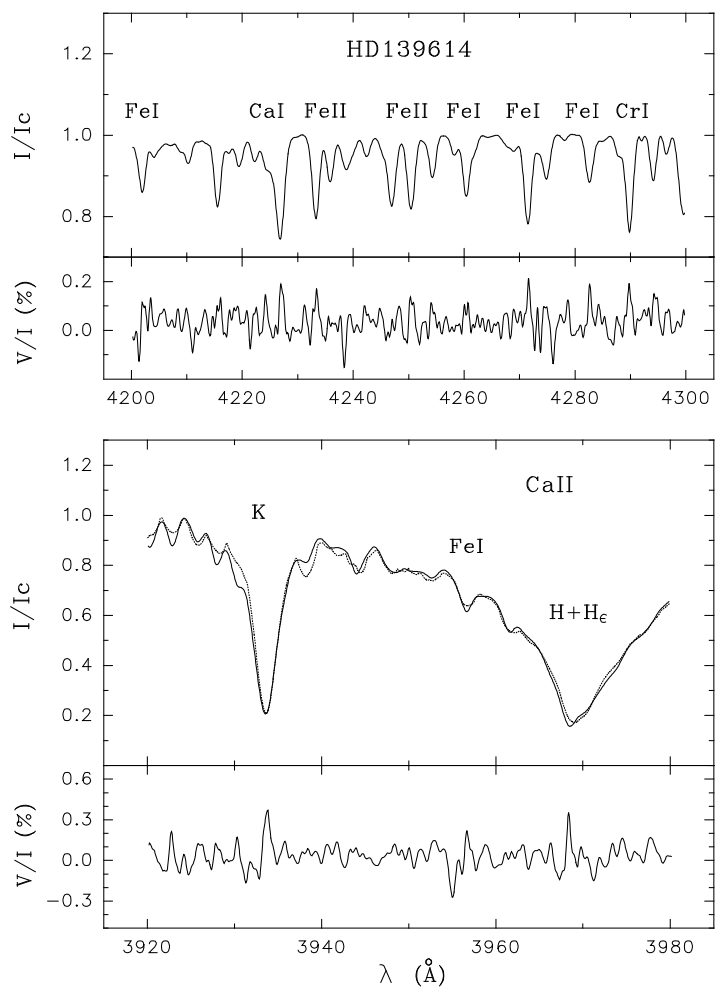

Fig. 1. Stokes $I$ and $V$ spectra of HD 139614 in two different spectral regions, observed in 2005. The synthetic photospheric spectrum in the lower panel is shown by the dotted line.

Stokes $V$ spectrum reveals circular polarisation signatures in the $\mathrm{Ca} \mathrm{K}$ and $\mathrm{H}$ components by displaying double-peaked features. The feature in the Fe I blend at $\lambda 3956.8 \AA$ is clearly visible in 2005 (Fig. 1), but absent in 2003. Similar circular polarisation signatures were also observed in $\mathrm{H} \beta, \mathrm{H} \gamma$, and $\mathrm{H} \delta$ lines. Thus, we assume that the magnetic field measured in 2003 (Hubrig et al. 2004) is mainly of CS origin. Our re-analysis of these observations excluding the spectral regions containing hydrogen lines leads to a magnetic field detection at the $3 \sigma$ level (see Table 2).

\subsubsection{HD 144432 (observed in 2003)}

Figure 5 demonstrates a notable difference in the $\mathrm{H} \beta$ profiles observed in 2003 and 2005. In 2003 this line was significantly
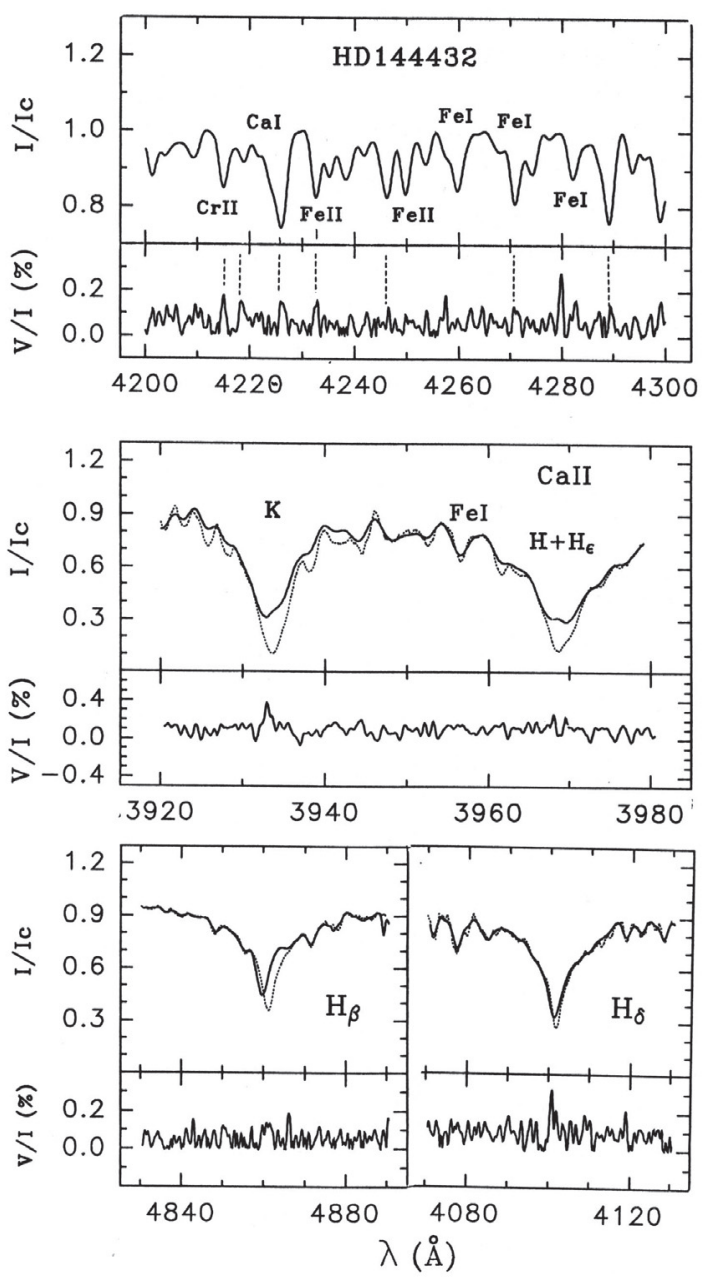

Fig. 2. Stokes $I$ and $V$ spectra of HD 144432 in four different spectral regions, observed in 2005. The dashed vertical lines in the upper panel indicate polarisation signatures corresponding to positions of stellar atmospheric lines. The synthetic photospheric spectrum is shown in the other panels by the dotted line.

deeper and displayed no emission. In contrast to the observations in 2005, no photospheric magnetic field was detected in 2003, thus a CS contribution to the photospheric spectrum was likely to be stronger during that measurement. It is possible that a larger 

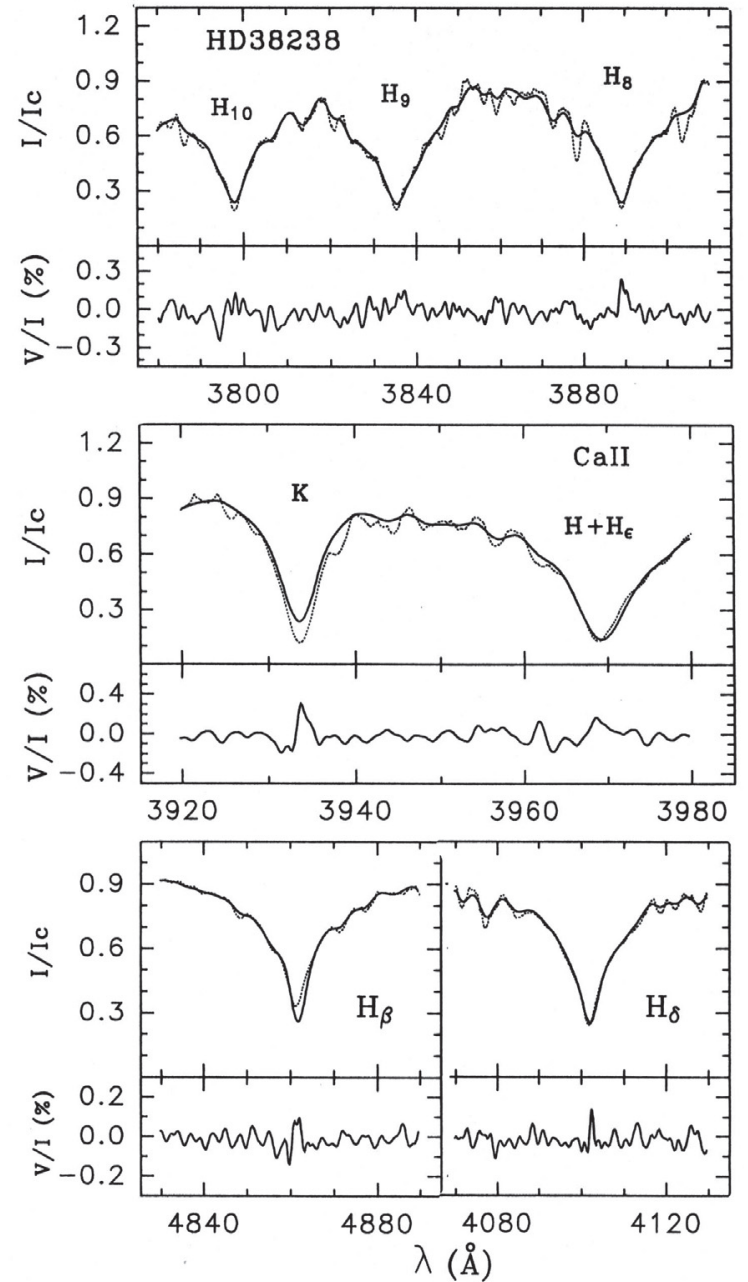

Fig. 3. Stokes $I$ and $V$ spectra of HD 38238 in four different spectral regions. The synthetic photospheric spectrum is shown in all panels by the dotted line.

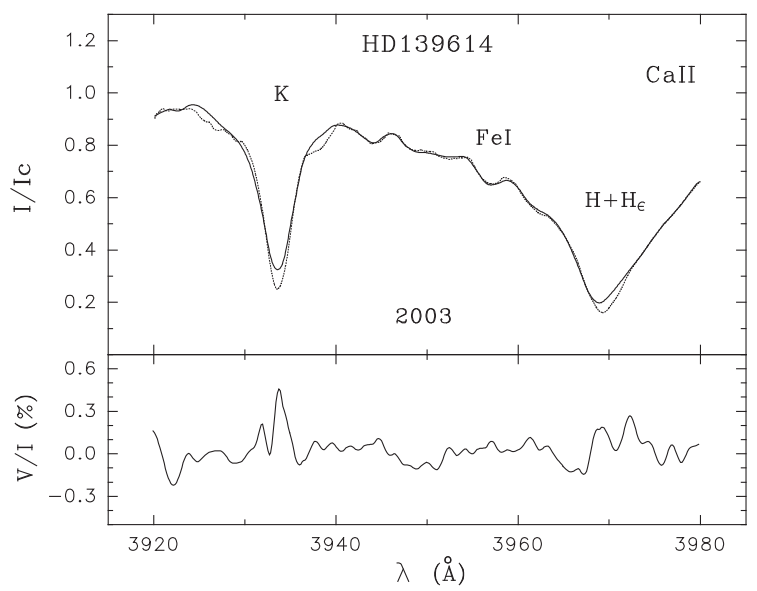

Fig. 4. Stokes $I$ and $V$ spectra of HD 139614 around the Ca II doublet. The synthetic photospheric spectrum is shown by the dotted line.

amount of dense outflowing gas was accumulated around the star. As a consequence, the circular polarisation signatures of the stellar magnetic field, which are clearly visible in 2005 , were likely distorted by CS material in the spectrum observed in 2003.

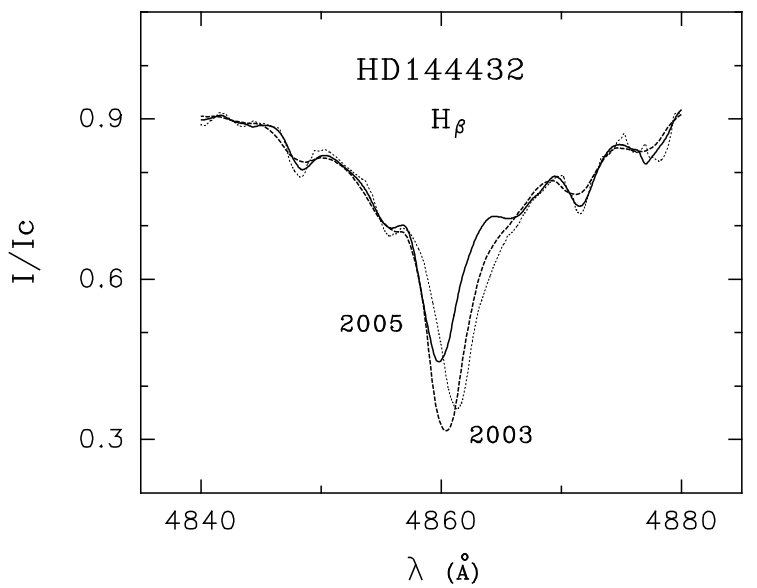

Fig. 5. The $\mathrm{H} \beta$ profiles of HD 144432 observed in 2003 (dashed line) and 2005 (solid line). The synthetic photospheric spectrum is shown by the dotted line.

\subsubsection{HD 144668}

As in the case of HD 38238, the first two spectropolarimetric observations of HD 144668 were carried out at lower spectral resolution $(R \sim 2000)$. The Stokes $V$ spectrum of this object, as well as the contribution of the CS matter to the spectrum, is also similar to those of HD 38238 (Fig. 6). We were able to measure a weak photospheric magnetic field only for MJD 52901.01 at a significance level of $4 \sigma\left(\left\langle B_{z}\right\rangle=166 \pm 40 \mathrm{G}\right)$.

\subsection{Group III}

\subsubsection{HD 31648}

HD 31648 is one of the hottest targets of the programme, demonstrating notable emissions in $\mathrm{H} \beta, \mathrm{H} \gamma$, and $\mathrm{H} \delta$ lines that indicate the presence of a significant stellar wind. The profiles of these lines are of P Cyg-type II (see Beals 1951) with very deep blueshifted absorptions (Fig. 7). Strong circular polarisation signatures associated with the CS component imply the presence of a considerable magnetic field in the outflowing gas. More evidence for the existence of the field is provided by the presence of notable polarisation signatures in the Ca II lines. The K profile of the doublet is complex and consists of two components: a blueshifted deep absorption and a red narrow one. The Ca II lines in the spectrum of HD 31648 are very likely formed at the base of the stellar wind, as well as in the accretion gaseous flow. Weak polarisation features are visible in the metallic lines. We conclude that a significant magnetic field is present in HD 31648 , but it is mostly of CS origin.

\subsubsection{HD 163296}

The Stokes $I$ spectrum of HD 163296 exhibits emission in the Balmer lines, similar to that of HD 31648. However, the Balmer line profiles are of P Cyg-type III with a secondary blueshifted emission component and a shallow absorption (Fig. 8). No circular polarisation signatures are visible in $\mathrm{H} \beta, \mathrm{H} \gamma$, and $\mathrm{H} \delta$, but such signatures are clearly observed in the lines of the Ca II doublet. The Ca II K line shows a two-component profile that is much wider than predicted by the synthetic photospheric spectrum. It is very likely that this line has a pure CS origin and forms in an envelope containing both outflowing and infalling gas. No indications of a photospheric magnetic field have been found in 

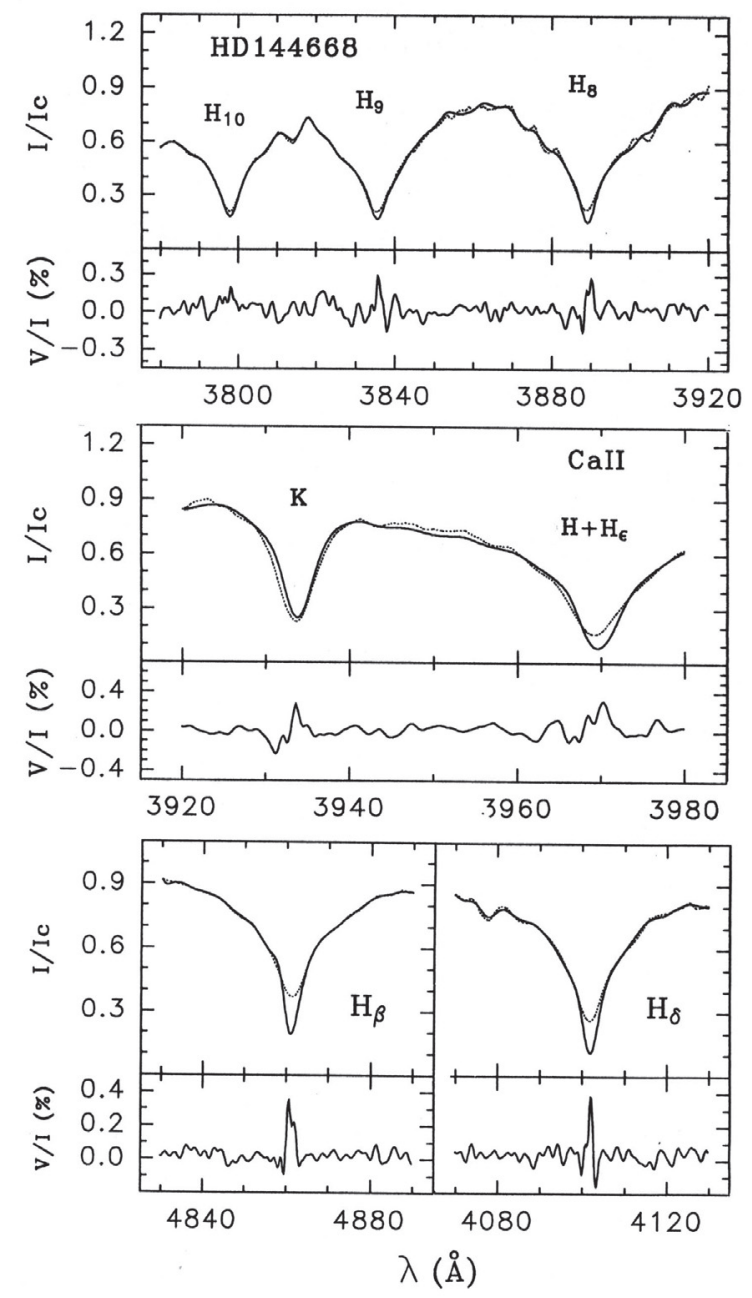

Fig. 6. HD 144668 on MJD 53120.25 (see caption of Fig. 3).

HD 163296. This object demonstrates the signature of the presence of a magnetic field only in the CS Ca II lines.

\subsubsection{HD 190073}

The most interesting results have been obtained for HD 190073, which is the third object of our programme with PCyg-type emissions in the Balmer lines. Three spectra of the star have been obtained on three different nights in 2005 (see Table 2). This allowed us to get an idea of the temporal behaviour of the object. The $\mathrm{H} \beta, \mathrm{H} \gamma$, and $\mathrm{H} \delta$ emissions are slightly variable with the PCyg structure being developed best on August 14, 2005 (see Fig. 9, bottom). This structure in $\mathrm{H} \beta$ looks to be of P Cygtype II with a rather weak blueshifted absorption. However, this absorption practically disappears in $\mathrm{H} \delta$. No circular polarisation signatures are found to be associated with these lines.

The most prominent spectral peculiarity of HD 190073 is the complex multi-component structure of the $\mathrm{Ca}$ II $\mathrm{H}$ and $\mathrm{K}$ absorption profiles. It consists of about 20 blueshifted components of different widths and depths (Pogodin et al. 2005, and references therein). The structure of the profiles is variable on timescales from years to decades, but it remains practically constant during a year. Our spectropolarimetry carried out at low spectral resolution allowed us to distinguish only the three strongest components. We did not detect any conspicuous profile variations. The mean Ca II spectrum is presented in the middle panel of
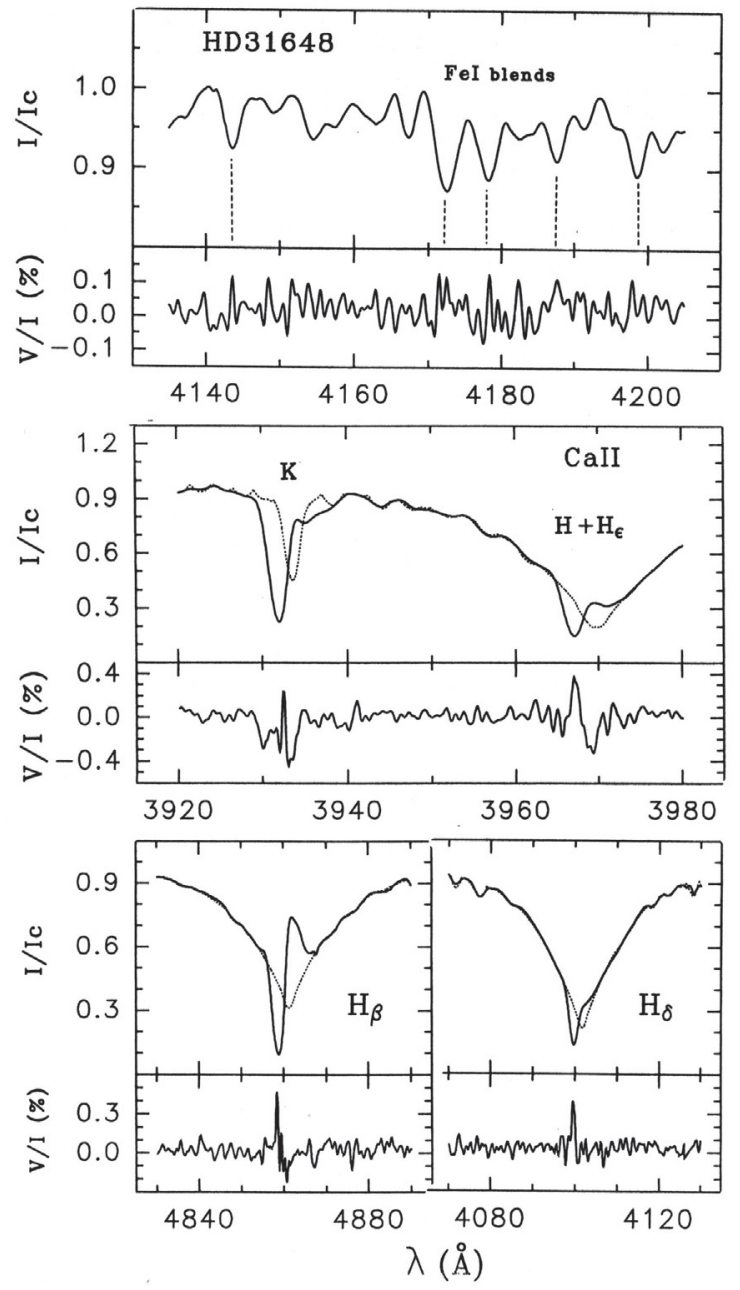

Fig. 7. HD 31648 (see caption of Fig. 2).
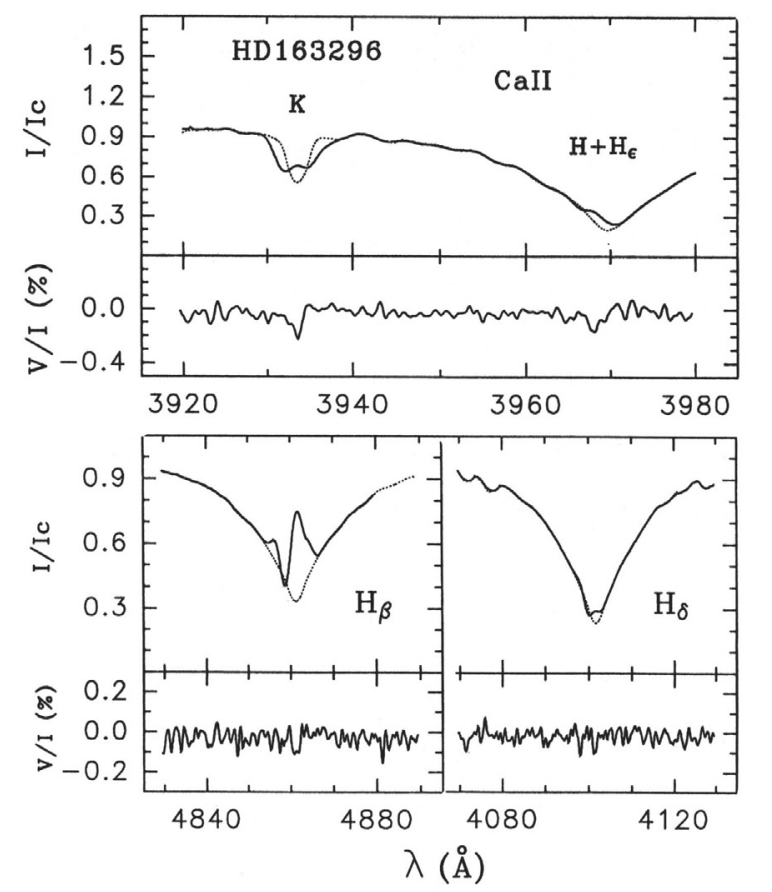

Fig. 8. Stokes $I$ and $V$ spectra of HD 163296 in three different spectral regions. The synthetic photospheric spectrum is shown in all panels by the dotted line. 

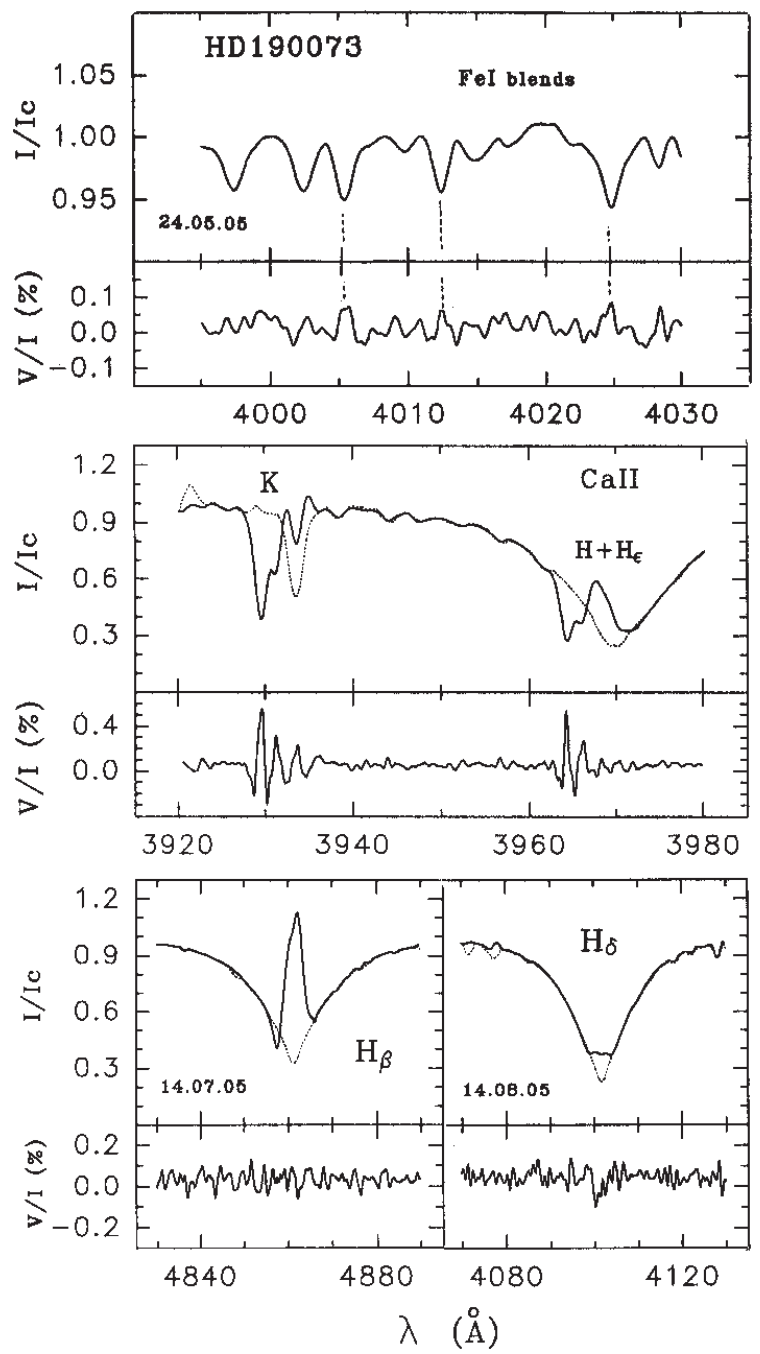

Fig. 9. Stokes $I$ and $V$ spectra of HD 190073 observed on May 24, 2005 (upper panel) and August 14, 2005 (bottom). The middle panel presents the mean Ca II spectrum. The synthetic photospheric spectrum is shown in all panels by the dotted line.

Fig. 9. Distinctive circular polarisation signatures are clearly visible, displaying three components in both $\mathrm{Ca}$ II $\mathrm{H}$ and $\mathrm{K}$ lines. Their positions coincide with the positions of three blueshifted components of the line profiles in the Stokes $I$ spectrum. The polarisation feature corresponding to the unshifted component, possibly of photospheric origin, has the lowest amplitude. The highest amplitude is observed in the most blueshifted component. This behaviour leads us to the conclusion that a direct relation must exist between the specific structure of the $\mathrm{Ca}$ II $\mathrm{H}$ and K line profiles observed in the spectrum of HD 190073 and the CS magnetic field.

A search for the photospheric magnetic field in HD 190073 and the study of its temporal behaviour revealed no significant polarisation signatures in the spectra observed on May 24 and 29, 2005. However, a weak magnetic field has been measured on the spectrum obtained on August 14, $2005\left(\left\langle B_{z}\right\rangle=\right.$ $-51 \pm 14 \mathrm{G})$.

\section{Discussion}

One of the important characteristics of the HAEBEs is the presence of a variable CS contribution to their stellar spectra, which strongly affects the results of spectropolarimetric studies of magnetic fields in this type of object. If the CS signatures are rather weak and observed only in a limited number of spectral lines, such as in the first members of the Balmer series, then it is possible to choose spectral regions that are not contaminated by the CS material and to measure the photospheric magnetic field with rather high precision (objects of group I). For the observations with a stronger CS contribution, the field detection becomes difficult, because of the problem identifying spectral regions with clean photospheric stellar lines (objects of group II). If CS lines become dominant in the spectrum of a Herbig star, then there is no way to measure the photospheric field, but it is possible to study the magnetic field present in the CS environment (objects of group III).

As a result of our magnetic-field investigation of seven HAEBEs, we make the following assertions:

- We conclude that previous discrepancies in estimations of magnetic fields for a few HAEBEs observed on different dates are likely the result of a variable CS contribution to the photospheric spectra of these objects.

- We have improved the accuracy of the photospheric magnetic-field determination of the two objects in group I, HD 139614 and HD 144432 (observed in 2005), to a significance level of about $7 \sigma$, instead of the previous $3 \sigma$ detections. This leaves little doubt about the presence of a magnetic field.

- The Stokes $V$ spectra of all seven programme targets demonstrate notable circular polarisation signatures, although for a few Herbig Ae stars, the effective magnetic field is too weak to be detected at a high significance level.

- We deduce that the measured magnetic fields of HD 31648 and HD 190073 (Hubrig et al. 2006) do not have a photospheric origin but have a mostly (HD 31648) or exclusively (HD 190073) CS origin.

- From the analysis of polarisation signatures of the CS magnetic field in the Herbig Ae stars HD 31648 and HD 144432, we conclude that in the Balmer lines the polarisation signatures are only observed in deep, blueshifted absorption components formed in a dense stellar wind in the region around the star.

- We found that the most sensitive indicator of the CS magnetic field in Herbig Ae stars is the Ca II doublet. Circular polarisation features corresponding to this doublet are observed in all programme targets of groups II and III. From the analysis of HD 31648 and HD 163296, we suggest that the magnetic field diagnosed in the Ca II lines is the one present in the CS matter in the vicinity of the stellar surface where the base of the stellar wind, as well as gaseous flows infalling onto the star, are likely to be located.

- The results of our study of HD 190073 have confirmed the magnetic nature of the well-known stable complex structure of absorption Ca II profiles in the spectrum of the object. The observations presented in Fig. 9 are in line with the hypothesis of Pogodin et al. (2005), who suggests that this structure is a result of a latitudinal stratification of the stellar wind governed by a global magnetic field with complex topology.

We would like to emphasise that the most effective strategy for future studies of magnetic fields of HAEBEs will be to carry out spectropolarimetric monitoring of these stars at different stages of their CS contribution to the photospheric spectrum. In the state of minimum CS distortion, the photospheric magnetic field is relatively easy to detect. When the CS spectrum becomes the most prominent, one has a good chance of studying the field in the CS environment. We also note that spectropolarimetric 
observations carried out at higher spectral resolution would allow us to analyse polarisation signatures in more detail and to use a considerably larger sample of spectral lines originating at different levels in the stellar atmosphere and the CS envelope. This should provide an opportunity to reconstruct the magnetic field topology from the stellar surface to the external CS envelope.

Acknowledgements. We would like to thank the referee, Richard Ignace, for his valuable comments.

\section{References}

Angel, J. R. P., \& Landstreet, J. D. 1970, ApJ, 160, L147

Bagnulo, S., Szeifert, T., Wade, G. A., et al. 2002, A\&A, 389, 191
Beals, C. S. 1951, in Publications of the Dominion Astrophysical Observatory Victoria, 9, 1

Böhm, T., Catala, C., Balona, L., \& Carter, B. 2004, A\&A, 427, 907

Catala, C., Böhm, T., Donati, J. F., \& Semel, M. 1993, A\&A, 278, 187

Donati, J. F., Semel, M., Carter, B. D., et al. 1997, MNRAS, 291, 658

Feigelson, E. D., Lawson, W. A., \& Garmire, G. P. 2003, ApJ, 599, 1207

Finkenzeller, U., \& Mundt, R. 1984, A\&AS, 55, 109

Glagolevskij, Yu. V. \& Chountonov, G. A. 2001, BSAO, 51, 88

Guenter, E. W. 1997, in Herbig-Haro flows and the birth of low mass stars (Kluwer Acad. Publ.), 465

Herbig, G. H. 1960, ApJ, 4, 337

Hubrig, S., Schöller, M., \& Yudin, R. V. 2004, A\&A, 428, L1

Hubrig, S., Yudin, R. V., Schöller, M., \& Pogodin, M. A. 2006, A\&A, 446, 1089

Ignace, R., \& Gayley, K. G. 2003, MNRAS, 341, 179

Piskunov, N. E. 1992, in Stellar Magnetism, S. Petersburg, Nauka, 92

Pogodin, M. A., Franco, G. A. P., \& Lopes, D. F. 2005, A\&A, 438, 239

Thé, P. S., de Winter, D., \& Pérez, M. R. 1994, A\&AS, 104, 315

Wade, G. A., Drouin, D., Bagnulo, S., et al. 2005, A\&A, 442, L31 